\title{
Clinical Utility Gene Card for: incontinentia pigmenti
}

\author{
Francesca Fusco ${ }^{1}$, Alessandra Pescatore ${ }^{1}$, Julie Steffann ${ }^{2}$, Ghislaine Royer ${ }^{2}$, Jean-Paul Bonnefont ${ }^{2}$ and Matilde \\ Valeria Ursini ${ }^{\star, 1}$
}

European Journal of Human Genetics (2013) 21, doi:10.1038/ejhg.2012.227; published online 10 October 2012

\section{DISEASE CHARACTERISTICS}

1.1 Name of the disease (synonyms)

Incontinentia pigmenti (IP); familial male-lethal type, BlochSulzberger syndrome; IP TYPE II; IP2.

\subsection{OMIM\# of the disease}

308300 .

1.3 Name of the analysed genes or DNA/chromosome segments NEMO/IKBKG, X Chomosome, Xq28.

\subsection{OMIM\# of the gene(s)}

300248 .

\subsection{Mutational spectrum}

IP is a rare X-linked genodermatosis, characterized by typical skin alterations, the hallmarks of the disease, and, in addition, by other neuroectodermal defects affecting the eyes, the nails, the hair, the teeth, and the central nervous system (CNS).

The clinical diagnosis of IP is based on the presence of dermatological lesions that develop in four successive, sometimes overlapping, characteristic stages that start shortly after birth with an inflammatory vesicular rash (stage1), followed by verrucous lesions (stage2). The third stage is marked by the appearance of a skin area displaying hyperpigmentation that at the fourth stage becomes patches of atrophic hypopigmented skin. In addition, IP females have heterogeneous and often severe clinical signs including ophthalmological (strabismus, cataracts, optic atrophy, retinal vascular pigmentary abnormalities, microphthalmia), odontological, (partial anodontia, delayed dentition, cone/peg-shaped teeth, impactions) and neurological defects (seizures, spastic paralysis, motor, and mental retardation, microcephaly). ${ }^{1}$

IP is a genomic disorder inherited as an X-linked dominant trait. IP is generally lethal in males while heterozygous females survive owing to functional mosaicism. ${ }^{1}$ All cases of IP are due to mutations in NEMO (nuclear-factor-kappa-B essential modulator)/IKBKG gene located in $\mathrm{Xq} 28$ region, and the mutation detection rate in IP is around $80 \%$. IKBKG, encodes the regulatory subunit of the IkB kinase complex required for nuclear factor- $\mathrm{kB}$ (NF-kB) activation. ${ }^{2,3}$

Mutations in different domains of protein may produce different effects on NF-kB activation by reducing or abolishing the response after stimulations. Noteworthy, some IKBKG hypomorphic mutations, affecting the zinc finger (ZF) domain of the NEMO protein and reducing but do not eliminating NF-kB activation, were found in surviving male patients. These males are affected by a different disease, named hypohidrotic ectodermal dysplasia-associated with severe immunodeficiency (EDA-ID HED-ID OMIM\#300291) or occasionally associated with osteopetrosis and lymphoedema (OL-EDA-ID)., ${ }^{4,5}$

The most frequent mutation in IP (70\%) is a recurrent exon 4_10 deletion (NEMOexon4_10del) due to non allelic homologous recombination that occurs between two repeats (MER67B) located in intron 3 and downstream exon 10, causing the removal of the entire genomic region from exon4 to $10.6,7$

Missense, nonsense, deletions, and insertions have been reported in addition to gross rearrangements. ${ }^{8}$ With the exception of a tract of cytosines in exon 10 that appears to be prone to mutations in IP/HED-ID, no mutational hotspots or common point mutations are seen.

To date, 53 different mutations (from large deletions to single amino-acid substitutions) affecting IKBKG have been reported: 7 gross deletions, 27 frameshift, 11 nonsense, four missense, one is an in-frame deletion of one codon, two are splice-site mutations, and one is a nonstop mutation. ${ }^{6-8}$

No evident genotype-phenotype correlation is apparent from comparison of patients with different loss-of-function mutations. The majority of mutations are 'private' to specific families. The rate of de novo mutations is about $65 \%$.

\subsection{Analytical methods}

Different strategies for IKBKG mutation screening procedures are currently applied on genomic DNA extracted from peripheral blood:

1. Long-range PCR using two specific primers able to detect the pathogenical IKBKG deletion (IKBKGexon4_10del) in the gene and able to discriminate it from the non pathogenical pseudogene deletion (PIKBKGexon4_10del). ${ }^{9,10}$ Indeed, a non-functional copy of the $I K B K G$ gene is located ( $99 \%$ identity with the gene) in the IP locus. ${ }^{11}$ No evidence of an involvement of the PIKBKG pseudogene in human diseases has so far been reported. The exon 4_10 deletion of PIKBKG is a benign variant in the control population. ${ }^{12}$ Those cases support the need to discriminate between deletions occurring in gene or in pseudogene to perform a correct molecular diagnosis of IP. ${ }^{9}$ A possible way to discriminate between IKBKG and PIKBKG deletions consists in testing the $\mathrm{X}$ inactivation pattern in white blood cells from female carriers. Conversely to PIKBKG deletions, IKBKG deletions are

\footnotetext{
${ }^{1}$ Institute of Genetics and Biophysics 'Adriano Buzzati-Traverso', IGB-CNR, Naples, Italy; 2Department of Genetics, Necker Enfants Malades Hospital Paris, Paris Descartes University, Paris, France

*Correspondence: Dr MV Ursini, Institute of Genetics and Biophysics 'Adriano Buzzati-Traverso', IGB-CNR, Via P. Castellino, 111, 80131 Naples, Italy. Tel: +39 081 6132262;

Fax: +39081 6132706; E-mail: ursini@igb.cnr.it
} 
almost consistently associated with a full $\mathrm{X}$ inactivation skewing, at least after one year of age. This test usually relies on the amplification of either a polymorphic CAG repeat in the Androgen receptor gene ${ }^{13}$ or a polymorphic CGG repeat in the FMR1 gene. ${ }^{14}$ It additionally enables to determine the parental origin of the rearranged chromosome, an useful information for genetic counselling purpose in apparently sporadic cases.

2. When no large deletion is identified in the gene, while both clinical picture and $\mathrm{X}$ inactivation skewing are highly suggestive of a IKBKG anomaly, a microrearrangement can be searched for, using direct sequencing of the coding regions with flanking intronic sequences of the IKBKG gene. If an intragenic mutation is detected, it is necessary to verify that the mutation has occurred in the gene and not in the pseudogene. This requires additional testing by long-range PCR amplification able to generate gene-specific and/or pseudogene-specific products, followed by PCR nested method and sequence analysis. ${ }^{6}$

3. When no point mutation is identified, a search for IKBKG genomic rearrangements different from the prevalent exon 4_10 deletion is performed by MLPA or quantitative real- time PCR using oligo primers distributed along the IP locus. A total of 26 primers for quantitative PCR, sequencing of breakpoint junction are used. Using such qPCR seven genomic deletions (35\%) were found in study of 20 patients who met diagnostic criteria for IP disease molecularly unsolved. ${ }^{8}$ This test is currently only used in research field, but may be used as a clinical test in the future. If genomic rearrangements, outside the IP locus, are suspected, highdensity array CGH can be performed.

\subsection{Analytical validation}

Parallel analysis of negative and positive controls. Direct sequencing of both DNA strands is performed. All mutations identified should be confirmed by a second, independent test (long-range PCR, quantitative real-time PCR, sequencing). Sequencing results are confirmed by re-sequencing using different sets of primers. It is recommended to confirm the segregation of the mutation in the parents. For potential missense mutations, protein assays and stability may be performed on a research basis using recombinant NEMO protein containing the mutation. Moreover, missense mutations should be in evolutionary conserved regions and they should be predicted by applicable software to be considered probably pathogenic.

\subsection{Estimated frequency of the disease}

(incidence at birth ('birth prevalence') or population prevalence. If known to be variable between ethnic groups, please report) Birth prevalence: 1:10000-1:20000.

\subsection{Diagnostic setting}

\begin{tabular}{lcc}
\hline & Yes & No \\
A. (Differential) diagnostics & $\bigotimes$ & $\square$ \\
B. Predictive testing & $\bigotimes$ & $\square$ \\
C. Risk assessment in relatives & $\bigotimes$ & $\square$ \\
D. Prenatal & $\bigotimes$ & $\square$
\end{tabular}

\section{Comment:}

Predictive testing in IKBKG should be considered on an individual case basis only, as long as no preventive treatment is available.

\section{TEST CHARACTERISTICS}

\begin{tabular}{|c|c|c|c|c|}
\hline & \multicolumn{2}{|c|}{ Genotype or disease } & \multirow{2}{*}{$\begin{array}{l}\text { A: True positives } \\
\text { B: False positives }\end{array}$} & \multirow{2}{*}{$\begin{array}{l}\text { C: False negative } \\
\text { D: True negative }\end{array}$} \\
\hline & Present & Absent & & \\
\hline \multicolumn{5}{|l|}{ Test } \\
\hline \multirow[t]{2}{*}{ Positive } & A & B & Sensitivity: & $A /(A+C)$ \\
\hline & & & Specificity: & $D /(D+B)$ \\
\hline \multirow[t]{2}{*}{ Negative } & $\mathrm{C}$ & $\mathrm{D}$ & Positive predictive value: & $A /(A+B)$ \\
\hline & & & Negative predictive value: & $\mathrm{D} /(\mathrm{C}+\mathrm{D})$ \\
\hline
\end{tabular}

\subsection{Analytical sensitivity \\ (proportion of positive tests if the genotype is present)}

Long-range PCR: $100 \%$ (only heterozygous IKBKGexon 4_10 deletion).

IKBKG sequencing: $>80 \%$ (heterozigous for IKBKG point mutation).

Quantitative PCR: 60\% heterozygous IKBKG deletion.

Comment: Quantitative PCR does not detect the point mutations in the gene nor other genomic alterations outside the IP locus. Depending on the technique and methods used in each laboratory, the sensitivity may vary.

It is recommended to scan SNP data bases periodically, to check for the identification of novel SNPs, prone to interfere with primer hybridization (http://www.ncbi.nlm.nih.gov/).

\subsection{Analytical specificity} (proportion of negative tests if the genotype is not present) Long-range PCR: $100 \%$, provided that the PCR specifically targets the IKBKG gene.

IKBKG sequencing: $>90 \%$. The main concern is the occasional detection of exonic variants of uncertain significance, of which the responsibility for the disease is often difficult to demonstrate.

Quantitative PCR: $>90 \%$ for heterozygous $I K B K G$ deletions.

\subsection{Clinical sensitivity}

(proportion of positive tests if the disease is present)

The clinical sensitivity depends on variable factors such as age or family history. Moreover, IP patients have heterogeneous clinical presentation. Indeed, while they have always-typical linear skin lesions (starting at birth and spontaneously evolving in four overlapping dermatological stages), they inconsistently exhibit ophthalmologic (strabismus, cataracts, optic atrophy, retinal vascular pigmentary abnormalities, microphthalmia), odontological (partial anodontia, delayed dentition, cone/peg-shaped teeth, impactions), and neurological defects (seizures, spastic paralysis, motor and mental retardation, microcephaly). The severity of these additional clinical signs is variable. . $^{1,6,15}$

\subsection{Clinical specificity}

(proportion of negative tests if the disease is not present)

Clinical specificity is around $100 \%$. The only (rare) pitfall consists in detecting the PIKBKG deletion, erroneously interpreted as the IKBKG deletion. The clinical specificity can be dependent on variable factors such as age or family history. In most cases, a detailed clinical assessment and skin biopsy will have been performed before genetic testing; therefore, presence of typically skin alterations represents not 
only a prerequisite to start genetic testing but also for the interpretation of $I K B K G$ variations of uncertain significance.

\subsection{Positive clinical predictive value}

(life-time risk to develop the disease if the test is positive).

On the basis of the studies of large pedigrees, most, if not all, patients appear to develop symptoms. Skin lesions are almost consistently found, tooth and eye anomalies are found in more than $50 \%$ cases, and a CNS involvement is present in $10-30 \%$ cases. ${ }^{1,6,15}$ For patients who are tested, and result positive for mutations, genetic counselling should be provided.

\subsection{Negative clinical predictive value}

(Probability not to develop the disease if the test is negative)

Assume an increased risk based on family history for a non-affected person. Allelic and locus heterogeneity may need to be considered.

Index case in that family had been tested:

Undetermined. We estimate that it is close to $100 \%$.

Index case in that family had not been tested:

Undetermined. We estimate that it is $>95 \%$.

It is notewhorty to mention that there is a low level risk for somatic mutations in $I K B K G$ that could cause IP-like skin features, escaping classical molecular diagnosis.

\section{CLINICAL UTILITY}

3.1 (Differential) diagnostics: The tested person is clinically affected (To be answered if in 1.10 'A' was marked)

\subsubsection{Can a diagnosis be made other than through a genetic test?}

\begin{tabular}{ll}
\hline No & $\square$ (continue with 3.1.4) \\
Yes $\quad$ Clinically & Imaging \\
Endoscopy & $\square$ \\
Biochemistry & $\square$ \\
Electrophysiology & $\square$ \\
Other & Highly experienced dermatologist may be able to diag- \\
(please describe) & nose the IP, and the information from skin biopsy can \\
& help to complete the diagnosis.
\end{tabular}

\subsubsection{Describe the burden of alternative diagnostic methods to the patient}

IP disorder can be diagnosed clinically, but not solely, using the revised criteria for classification of IP which established that the affected females have a history of perinatal blistering and at least one of the other stages of skin lesions.

Clinical diagnosis may include: nervous system exam for seizures, spastic paresis, motor and mental retardation, microcephaly, ocular defects, dental defects, hair defects, and nail defects taking a family pedigree and clinical assessment by a clinical geneticist or other physician familiar.

Skin biopsy is painful and will not specify the underlying genetic defect.

\subsubsection{How is the cost effectiveness of alternative diagnostic methods to be judged?}

Low. In our experience, considering a IP diagnosis ineluctably results in request for genetic testing.
3.1.4 Will disease management be influenced by the result of a genetic test?

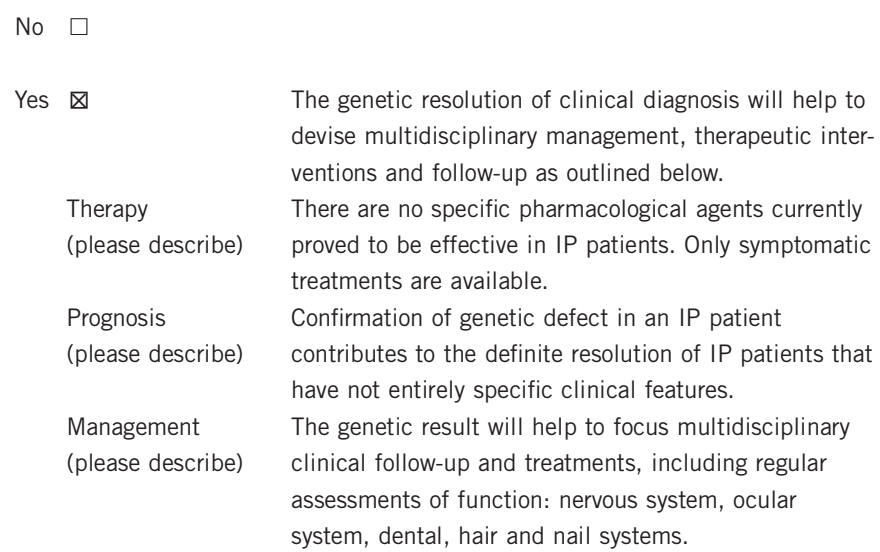

3.2 Predictive setting: The tested person is clinically unaffected but carries an increased risk based on family history

(To be answered if in 1.10 'B' was marked)

\subsubsection{Will the result of a genetic test influence lifestyle and prevention?}

If the test result is positive (please describe)

Identification of a IKBKG mutation allows carriers to make informed reproductive decisions, which take into account the risk of having an IP-affected child.

A woman with a mutation may decrease her risk of having an IP child affected with IP by taking advantage of prenatal diagnosis oocyte donation, adoption, and so on. Preimplantation genetic diagnosis (PGD) is possible but particular technical difficulties exist for IP.

If the test result is negative (please describe)

Determining that a female patient is not a carrier can relieve the anxiety related to genetic risk and allow for confident family planning.

3.2.2 Which options in view of lifestyle and prevention does a person at-risk have if no genetic test has been done (please describe)? No special options; prevention is not possible.

3.3 Genetic risk assessment in family members of a diseased person (To be answered if in 1.10 ' $\mathrm{C}$ ' was marked)

3.3.1 Does the result of a genetic test resolve the genetic situation in that family?

Yes, it confirms the mode of inheritance and is the prerequisite for genetic risk assessment in relatives.

3.3.2 Can a genetic test in the index patient save genetic or other tests in family members?

Yes, when the IKBKG mutation is shown to have occurred de novo in the proband.

\subsubsection{Does a positive genetic test result in the index patient enable a predictive test in a family member?}

Yes, a positive test in a female allows identifying her mother as a carrier if the mutation is not a de novo mutation. We personally recommend to test also for asymptomatic parents and other female relatives, both to determine the precise inheritance pattern in the 
family but also to advise the parents about their potential IP risk Indeed, even if a IKBKG mutation is usually 'fully' penetrant, IP signs can sometimes be underdiagnosed when clinical picture is restricted to scars of skin lesions.

\subsection{Prenatal diagnosis}

(To be answered if in 1.10 'D' was marked)

\subsubsection{Does a positive genetic test result in the index patient enable a prenatal diagnosis?}

All females with a IKBKG mutation can be offered a prenatal diagnosis.

\section{IF APPLICABLE, FURTHER CONSEQUENCES OF TESTING}

Please assume that the result of a genetic test has no immediate medical consequences. Is there any evidence that a genetic test is nevertheless useful for the patient or his/her relatives? (Please describe)

Yes. It is advised to confirm IP carrier status in affected mother.

Although there is no cure for IP, the diagnosis helps to follow appropriate physical, cognitive, and behavioural management of the affected individual.

Yes, genetic testing is the gold standard for confirmation of the diagnosis and the mode of inheritance, helps to avoid unnecessary and invasive diagnostic procedures. It allows prognostic evaluations and is the prerequisite for prenatal testing, PGD, and genetic risk estimation of relatives.

Molecular confirmation of the diagnosis will limit unnecessary further aetiological investigations, which can often be invasive and unpleasant.

Many parents feel guilty, and may be relieved after a genetic diagnosis is obtained. Parents also find encouragement and support in dealing with daily anxieties and difficulties by becoming members of clubs and associations that welcome affected families.

A molecular diagnosis enables a female carrier of mutation to make informed reproductive decisions.

\section{CONFLICT OF INTEREST}

The authors declare no conflict of interest.

\section{ACKNOWLEDGEMENTS}

This work was supported by Telethon (grant\#GGP08125) to MVU and by Fondazione 'Roma-Terzo Settore' to MVU. AP is supported by a Postdoctoral fellowship from the Association Incontinentia Pigmenti France (IPF). The authors wish to thank the patients, their families and patient associations: Italian I.P.ASS.I. Onlus (www.incontinentiapigmenti.it), the Incontinentia Pigmenti International Foundation (IPIF) (www.ipif.org), and the Incontinentia Pigmenti France (http://www.incontinentiapigmenti.fr).

1 Scheuerle A, Ursini MV: Incontinentia Pigmenti; in Pagon RA, Bird TD, Dolan CR, Stephens K (eds) GeneReviews. University of Washington: Seattle (WA), 2010; pp 1993-1999.

2 Hayden MS, Ghosh S: Signaling to NF-kappaB. Genes Dev 2004; 18: 2195-2224.

3 Nelson DL: NEMO, NFkappaB signaling and Incontinentia Pigmenti. Curr Opin Genet Dev 2006; 16: 282-288.

4 Doffinger R, Smahi A, Bessia C et al: X-linked anhidrotic ectodermal dysplasia with immunodeficiency is caused by impaired NF-kappa-B signaling. Nat Genet 2001; 27: $277-285$.

5 Zonana J, Elder ME, Schneider LC et al: A novel X-linked disorder of immune deficiency and hypohidrotic ectodermal dysplasia is allelic to incontinentia pigmenti and due to mutations in IKK-gamma (NEMO). Am J Hum Genet 2000; 67: 1555-1562.

6 Fusco F, Bardaro T, Fimiani G et al: Molecular analysis of the genetic defect in a large cohort of IP patients and identification of novel NEMO mutations interfering with NF-kappaB activation. Hum Mol Genet 2004; 13: 1763-1773.

7 Fusco F, Pescatore A, Bal E et al: Alterations of the IKBKG locus and diseases: an update and a report of 13 novel mutations. Hum Mutat 2008; 29: 595-604.

8 Fusco F, Paciolla M, Napolitano F et al: Genomic architecture at the Incontinentia Pigmenti locus favours de novo pathological alleles through different mechanisms. Hum Mol Genet 2012; 21: 1260-1271.

9 Bardaro T, Falco G, Sparago A et al: Two cases of misinterpretation of molecular results in incontinentia pigmenti, and a PCR-based method to discriminate NEMO/IKKgamma gene deletion. Hum Mutat 2003; 21: 8-11.

10 Steffann J, Raclin V, Smahi A et al: A novel PCR approach for prenatal detection of the common NEMO rearrangement in incontinentia pigmenti. Prenat Diagn 2004; 24: 384-388.

11 Aradhya S, Bardaro T, Galgoczy $\mathrm{P}$ et al: Multiple pathogenic and benign genomic rearrangements occur at a $35 \mathrm{~kb}$ duplication involving the NEMO and LAGE2 genes. Hum Mol Genet 2001a; 10: 2557-2567.

12 Fusco F, Paciolla M, Pescatore A et al: Microdeletion/duplication at the Xq28 IP locus causes a de novo IKBKG/ NEMO/IKKgamma exon4 10 deletion in families with Incontinentia Pigmenti. Hum Mutat 2009; 30: 1284-1291.

13 Allen RC, Zoghbi HY, Moseley AB et al: Methylation of Hpall and Hhal sites near the polymorphic CAG repeat in the human androgen-receptor gene correlates with $X$ chromosome inactivation. Am J Hum Genet 1992; 51: 1229-1239.

14 Houdayer C, Lemonnier A, Gerard M et al: Improved fluorescent PCR-based assay for sizing CGG repeats at the FRAXA locus. Clin Chem Lab Med 1999; 37: 397-402.

15 Hadj-Rabia S, Froidevaux D, Bodak N et al: Clinical study of 40 cases of incontinentia pigmenti. Arch Dermatol 2003; 139: 1163-1170. 